\title{
HAWK - Hybrid Question Answering using Linked Data
}

\author{
Ricardo Usbeck ${ }^{1}$, Axel-Cyrille Ngonga Ngomo ${ }^{1}$, Lorenz Bühmann ${ }^{1}$, and Christina \\ Unger $^{2}$ \\ 1 University of Leipzig, Germany \\ \{usbeck, ngonga\}@informatik.uni-leipzig.de \\ 2 University of Bielefeld, Germany \\ cungerdcit-ec.uni-bielefeld.de
}

\begin{abstract}
The decentral architecture behind the Web has led to pieces of information being distributed across data sources with varying structure. Hence, answering complex questions often required combining information from structured and unstructured data sources. We present HAWK, a novel entity search approach for Hybrid Question Answering based on combining Linked Data and textual data. The approach uses predicate-argument representations of questions to derive equivalent combinations of SPARQL query fragments and text queries. These are executed so as to integrate the results of the text queries into SPARQL and thus generate a formal interpretation of the query. We present a thorough evaluation of the framework, including an analysis of the influence of entity annotation tools on the generation process of the hybrid queries and a study of the overall accuracy of the system. Our results show that HAWK achieves 0.68 respectively $0.61 \mathrm{~F}$-measure within the training respectively test phases on the Question Answering over Linked Data (QALD-4) hybrid query benchmark.
\end{abstract}

\section{Introduction}

Recent advances in question answering (QA) over Linked Data provide end users with more and more sophisticated tools for querying linked data by expressing their information need in natural language $[16,18,19]$. This allows access to the wealth of structured data available on the Semantic Web also to non-experts. However, a lot of information is still available only in textual form, both on the Document Web and in the form of labels and abstracts in Linked Data sources [9]. Therefore, a considerable number of questions can only be answered by using hybrid question answering approaches, which can find and combine information stored in both structured and textual data sources [21].

In this paper, we present HAWK, the (to best of our knowledge) first full-fledged hybrid QA framework for entity search over Linked Data and textual data. Given an input query $q$, HAWK implements an 8-step pipeline, which comprises 1) part-of-speech tagging, 2) detecting entities in $q, 3$ ) dependency parsing and 4) applying linguistic pruning heuristics for an in-depth analysis of the natural language input. The results of these first four steps is a predicate-argument graph annotated with resources from the Linked Data Web. HAWK then 5) assign semantic meaning to nodes and 6) generates basic triple patterns for each component of the input query with respect to a multitude of 
features. This deductive linking of triples results in a set of SPARQL queries containing text operators as well as triple patterns. In order to reduce operational costs, 7) HAWK discards queries using several rules, e.g., by discarding not connected query graphs. Finally, 8) queries are ranked using extensible feature vectors and cosine similarity.

Our main contributions can be summarized as follows:

- We present the first QA framework tackling hybrid question answering;

- HAWK analyses input queries based on predicate-argument trees to deeply understand and match semantic resources;

- Our framework is generic as it does not rely on templates. It is thus inherently able to cover a wide variety of natural language questions as well as knowledge bases with various topologies;

- The modular architecture of HAWK allows simple exchanging of pipeline parts to enhance testing and deployment;

- Our evaluation suggests that HAWK is able to achieve f-measures of 0.61 on rather small training datasets.

The rest of the paper is structured as follows: Afterwards, our methodology is explained in detail in Section 2. HAWK's performance and the influence of entity annotation systems is evaluated in Section 3. Section 4 discusses related work. Finally, we conclude in Section 5. Additional information can be found at our project home page http://aksw.org/Projects/HAWK.html.

\section{Method}

In the following, we describe the architecture and methodology of HAWK. We explain our approach by using the following running example: Which recipients of the Victoria Cross died in the Battle of Arnhem? While this question cannot be answered by using solely DBpedia or Wikipedia abstracts, combining knowledge from DBpedia and Wikipedia abstracts allows deriving an answer to this question. More specifically, DBpedia allows to retrieve all recipients of the Victoria Cross using the triple pattern ?uri dbo: award dbr:Victoria_Cross .

In order to find out whether the returned resources died in the Battle of Arnhem, the free text abstract of those resources need to be checked. For example, the abstract for John Hollington Grayburn contains the following information: 'he went into action in the Battle of Arnhem [...] but was killed after standing up in full view of a German tank'.

Figure 1 gives an overview of the architecture of HAWK. In the following we describe the depicted steps in more details.

\subsection{POS-Tagging}

A large number of frameworks have been developed for these purposes over the last years. We rely on clearNLP [3] which is based on transition-based dependency parsing. Regarding our running examples the following POS-tags are generated: Which (WDT)

recipients (NNS) of(IN) the(DT) Victoria(NNP) Cross(NNP)

died(VBN) in(IN) the (DT) Battle(NNP) of (IN) Arnhem(NNP)? 


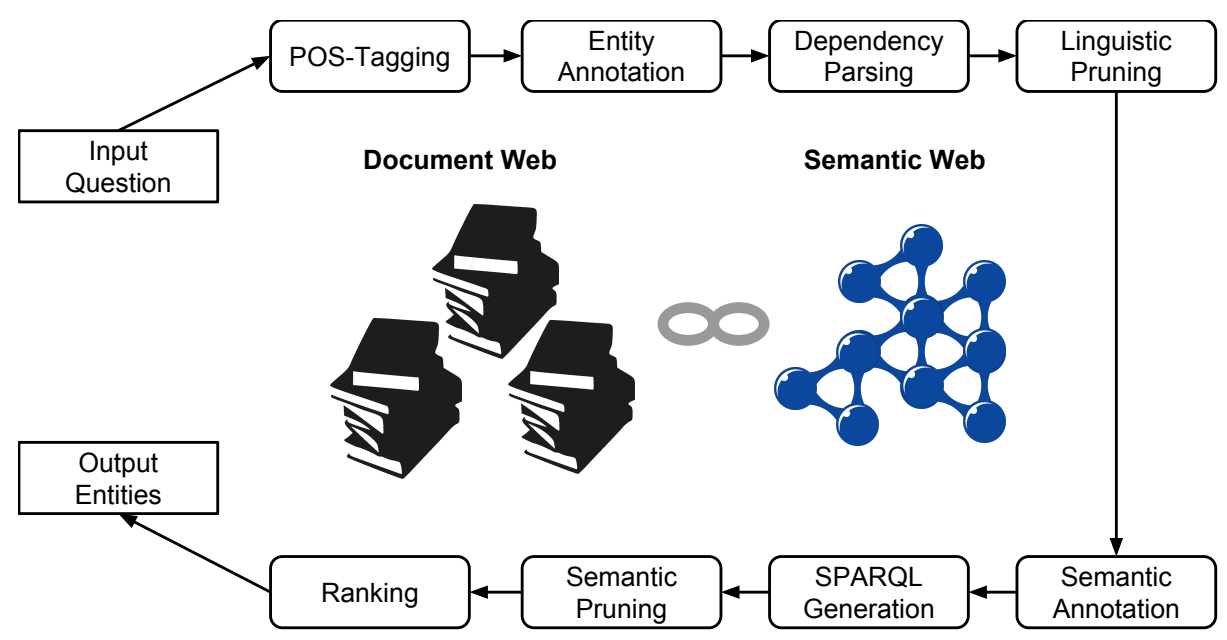

Fig. 1: Architectural overview of HAWK.

\subsection{Entity Annotation}

HAWK identifies named entities and tries to link them to semantic entities from the underlying knowledge base, in our case DBpedia 3.9, via well-established entity annotation tools:

- Wikipedia Miner [14] is based on different facts like prior probabilities, context relatedness and quality, which are then combined and tuned using a classifier.

- DBpedia Spotlight [13] was published in 2011. This tool combines named entity recognition and disambiguation based on DBpedia.

- TagMe 2 [6] is based on a directory of links, pages and an inlink graph from Wikipedia. The approach recognizes entities by matching terms with Wikipedia link texts and disambiguates the match using the in-link graph and the page dataset.

- FOX [17] has been introduced 2014 as an ensemble learning-based approach combining several state-of-the-art named entity recognition approaches. The FOX framework outperforms the current state of the art entity recognizer and relies on the entity linking tool AGDISTIS [22].

Additionally, we implemented two artificial spotters for evaluation:

- Union is a spotter that combines the result sets of the above introduce spotters and returns thus a superset of all spotters.

- Optimal will spot all entities from the gold standard to be able to ignore spotting influences in the following steps of the pipeline.

For our running example, an optimal spotter identifies Victoria_Cross and Batt le_of Arnhem as resources form DBpedia. HAWK annotates the POS-tag ADD to it. The influence of the entity annotation module is evaluated in Section 3. 


\subsection{Dependency Parsing}

HAWK performs noun phrase detection for semantically meaningful word groups not yet recognized by the entity annotation system. This detection reuses the above mentioned POS-tagger. Input tokens will be combined following linguistic heuristics derived from the benchmark questions, and their POS-tag is changed to CNN. The full algorithm is depicted in Algorithm 1. HAWK's modular structure allows for an easy exchange of the POS-tagger or dependency parser.

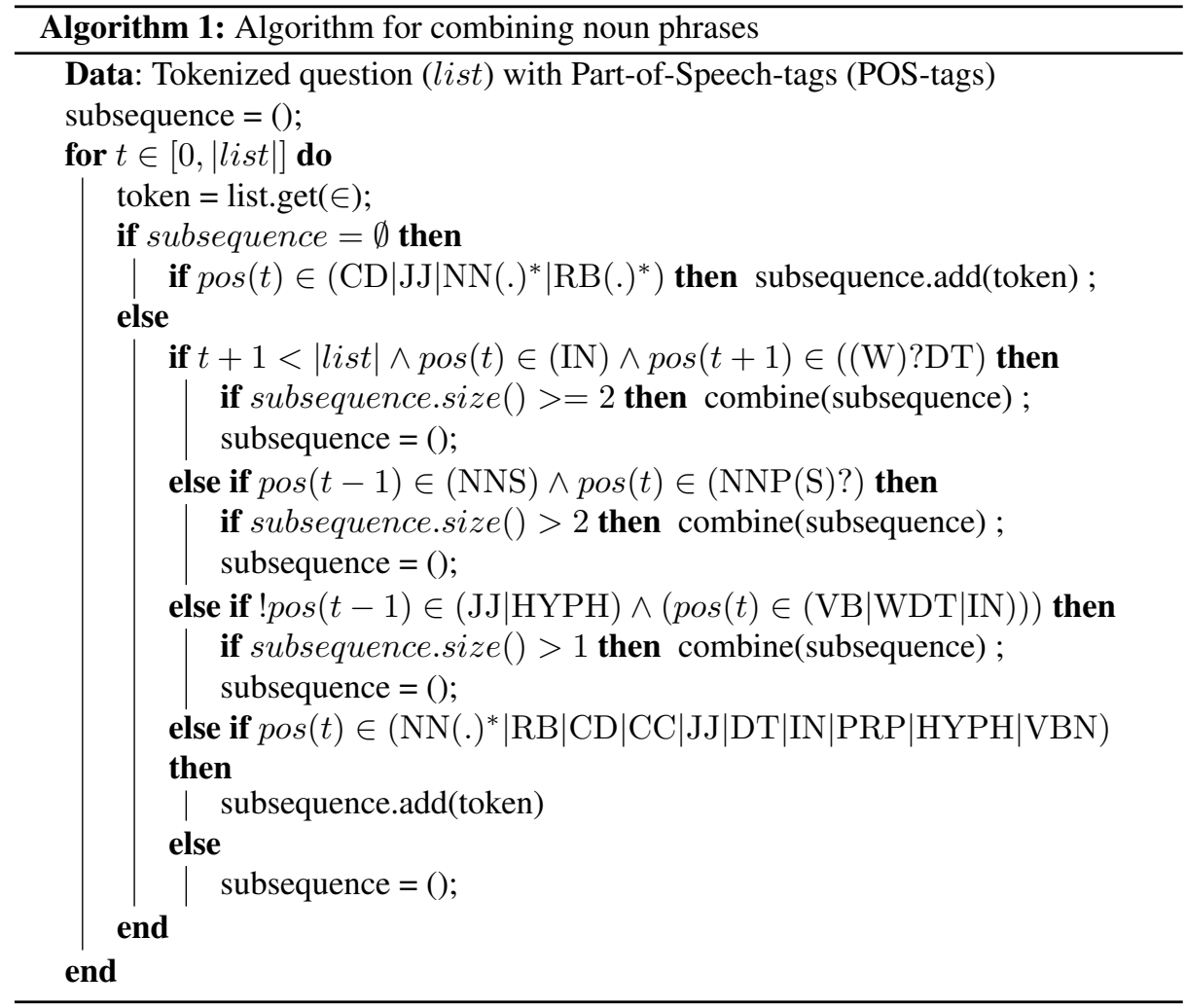

Subsequently, in order to capture linguistic and semantic relations, HAWK parses the query using dependency parsing and semantic role labeling [3]. The generated predicate-argument tree is directed, acyclic and all its nodes contain their POS-tags as well as their labels, see Figure 2.

\subsection{Linguistic Pruning}

The natural language input can contain tokens that are meaningless for retrieving the target information or even introduce noise in the process. HAWK therefore prunes nodes from the predicate-argument tree based on their POS-tags, e.g., deleting all DET nodes, interrogative phrases such as Give me or List, and auxiliary tokens such as did. Algorithm 2 details the algorithm for removing nodes. Figure 3 depicts the predicateargument tree obtained for our running example after pruning. 

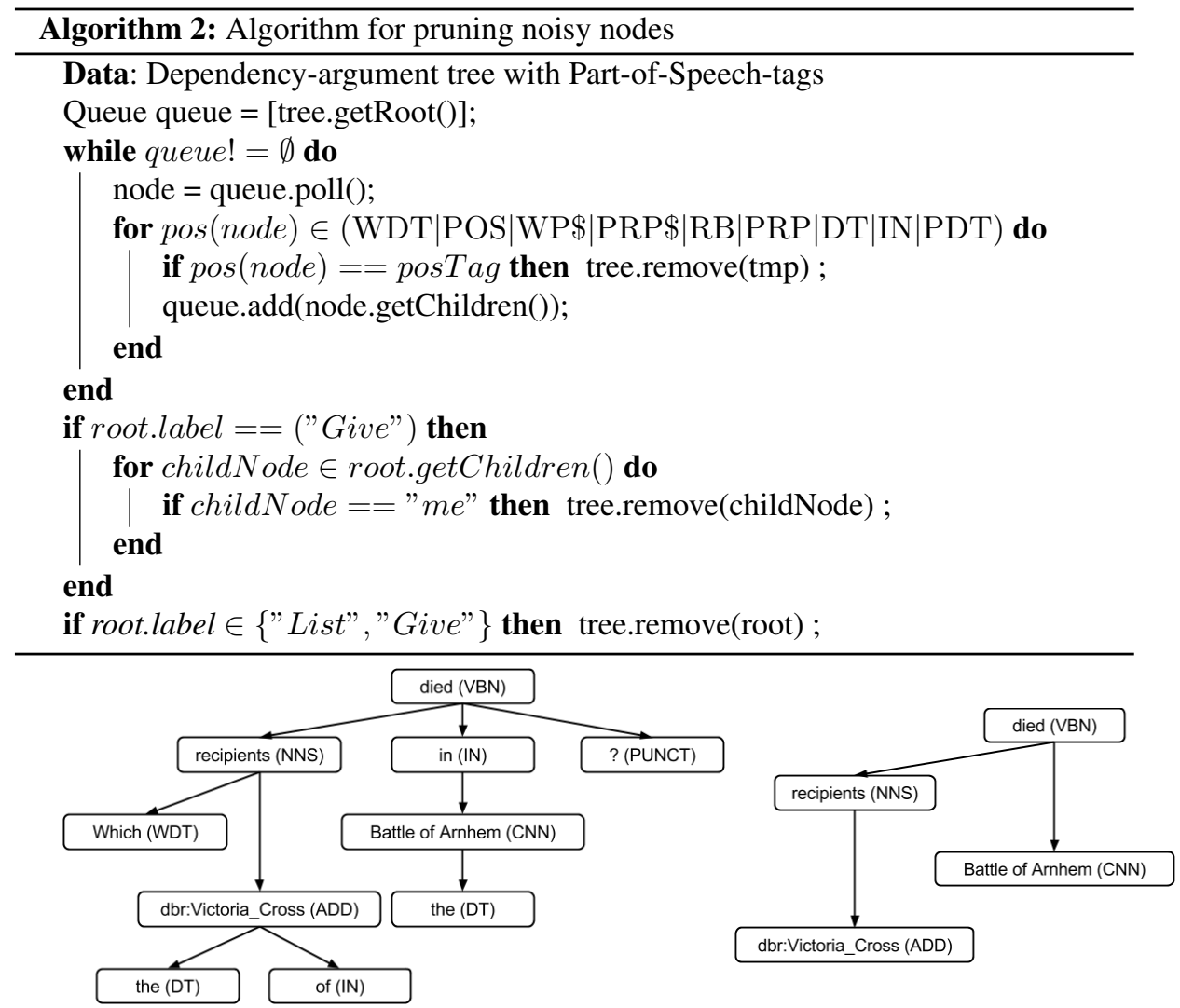

Fig. 2: Predicate-argument tree for the example question 'Which recipients of the Victoria Cross died in the Battle of Arnhem?'

Fig. 3: Tree after pruning.

\subsection{Semantic Annotation}

After linguistic pruning, HAWK annotates each node in the tree with possible concepts from the knowledge base and its underlying ontology. To this end, our framework uses information about possible verbalizations of ontology concepts, based on both rdfs: label information from the ontology itself and (if available) verbalization information contained in lexica. In general, such lexica offer a range of lexical variants beyond the labels present in DBpedia. For example, for the property spouse, the DBpedia English lexicon ${ }^{3}$ provides the noun entries 'wife' and 'husband' as well as the verb entry 'to marry'.

HAWK now tries to match each node label to a class or property from the DBpedia ontology using fuzzy string matching. Moreover, HAWK follows intuitions used in [18] to lower the number of annotations avoiding additional computational effort. In particular, we consider the POS-tag of nodes to determine the type of the target reference:

${ }^{3}$ https://github.com/cunger/lemon.dbpedia 
- nouns correspond to object type properties and classes

- verbs correspond to object type properties

- question words (e.g., who or where) correspond to classes (e.g., Person or Place)

Afterwards, HAWK ranks properties according to their prominence score in the knowledge base and returns only the top n properties. If the search does not retrieve any annotations, we additionally ask the lemmata of the node label and repeat the above described process.

Considering our running example, Table 1 presents possible matches for the nodes died (VB) and recipients (NNS). After this step, either a node is annotated with a reference from the knowledge base, it is a disambiguated resource or it will be lead to a full-text lookup to be resolved to a knowledge base resource.

\begin{tabular}{l}
\hline Annotation \\
\hline died dbo: deathplace, dbo: deathdate \\
recipients dbo: award \\
Table 1: Annotations of nodes from running example.
\end{tabular}

\subsection{Generating SPARQL Queries}

The core of HAWK is the generation of SPARQL queries from annotated and pruned predicate-argument trees. To capture the full semantics of an input question, HAWK traverses the predicated-argument tree in a pre-order walk to reflect the empirical observation that i) related information are situated close to each other in tree and ii) information are more restrictive from left to right. This breadth-first search visits each nodes and generates several possible triple patterns based on the number of annotations and the POS-tag itself. That is, for each node a set of SPARQL query patterns is generated following the rules depicted in Table 3 w.r.t. ontology type information, e.g., a variable bound to the class Place will not have an outgoing predicate birthPlace.

Using this approaches allows HAWK to be independent of SPARQL templates and to work on natural language input of any length and complexity. Each pattern contains at least one variable from a pre-defined set of variables, i.e., ?proj for the resource projection variable, ? const for resources covering constraints related to the projection variable as well as a variety of variables for predicates to inspect the surrounding of elements in the knowledge base graph. Table 2 shows generated triple patterns for parts of the example query.

During this process, each iteration of the traversal appends the generated patterns to each of the already existing SPARQL queries. This combinatorial effort results in covering every possible SPARQL graph pattern given the predicate-argument tree. 


\begin{tabular}{ll}
\hline Node Type & Query Fragment \\
\hline CNN & ?proj text:query ('Battle of Arnhem') \\
& ?const text:query ('Battle of Arnhem') \\
\hline Verb & ?proj dbo:deathPlace ?const \\
& ?const dbo: deathPlace ?proj \\
\hline
\end{tabular}

Table 2: Generated triple patterns for running example.

\begin{tabular}{|c|c|}
\hline Node POS-tag and non-empty annotat & Query Fragment \\
\hline $\begin{array}{l}\operatorname{VB}(.)^{*} \\
\operatorname{VB}(.)^{*} \\
\operatorname{VB}(.)^{*} \\
\mathrm{NN}(.)^{*} \mid \mathrm{WRB} \\
\mathrm{NN}(.)^{*} \mid \mathrm{WRB} \\
\mathrm{NN}(.)^{*} \mid \mathrm{WRB} \\
\mathrm{NN}(.)^{*} \mid \mathrm{WRB} \\
\mathrm{NN}(.)^{*} \mid \mathrm{WRB} \\
\mathrm{WP} \\
\mathrm{WP} \\
\text { all }\end{array}$ & $\begin{array}{l}\text { ?proj Annotation ?const. } \\
\text { ?const Annotation ?proj. } \\
\text { ?const ?proot ?proj. } \\
\text { ?proj Annotation ?const. } \\
\text { ?const Annotation ?proj. } \\
\text { ?proj a Annotation. } \\
\text { ?const a Annotation. } \\
\text { ?const text:query (node label) } \\
\text { ?const a Annotation. } \\
\text { ?proj a Annotation. } \\
\text { ignore }\end{array}$ \\
\hline Node POS-tag and empty annotations & Query Fragment \\
\hline $\begin{array}{l}\mathrm{CNN}\left|\mathrm{NNP}(.)^{*}\right| \mathrm{JJ} \mid \mathrm{CD} \\
\mathrm{CNN}\left|\mathrm{NNP}(.)^{*}\right| \mathrm{JJ} \mid \mathrm{CD} \\
\mathrm{VB}(.)^{*} \\
\mathrm{VB}(.)^{*} \\
\mathrm{ADD} \\
\mathrm{ADD} \\
\text { ADD } \\
\text { ADD } \\
\mathrm{NN} \mid \mathrm{NNS} \\
\mathrm{NN} \mid \mathrm{NNS} \\
\text { all }\end{array}$ & $\begin{array}{l}\text { ?proj text:query (node label) } \\
\text { ?const text:query (node label) } \\
\text { ?proj text:query (node label) } \\
\text { ?const text:query (node label) } \\
\text { ?proj ?pbridge nodeURI. } \\
\text { FILTER (?proj IN (nodeURI) } \\
\text { ?proj text:query (node label) } \\
\text { ?const text:query (node label) } \\
\text { ?proj text:query (node label) } \\
\text { ?const text:query (node label) } \\
\text { ignore node }\end{array}$ \\
\hline
\end{tabular}

Table 3: Triple pattern for generating SPARQL queries while traversal.

\subsection{Semantic Pruning of SPARQL Queries}

Producing the $\mathrm{n}$-fold-cross-product of possible pattern combinations generates a huge amount of SPARQL queries, most of which are semantically senseless. To effectively handle this large set of queries and reduce the computational effort, HAWK implements various methods for pruning:

- \#textfilter: HAWK can safely assume that SPARQL queries containing full-text lookups over more than one variable or containing more than two node labels do not yield semantically senseful information and thus discards such queries. 
- \#unbound triple pattern: SPARQL queries containing more than one triple pattern of the form ?varx ?vary ?varz or one such triple pattern and only text searches, lead to a traversal of large parts of the knowledge base graph and high computational effort.

- Unconnected query graph: SPARQL query graphs which are not connected from cartesian products are pruned for the sake of runtime and their lack of semantics.

- Cyclic triple: Queries containing edges of the form ?s $\langle$ http://xyz $\rangle$ ?०. ?o <http://xyz> ?s or ?s <http://xyz> ?o. ?s <http://abc> ?० are also removed.

- Missing projection variable: The before mentioned traversal and SPARQL generation process can produce SPARQL queries without triple patterns containing the projection variable. These queries are also removed from the set of queries.

- Disjointness: Also SPARQL queries with triple patterns violating disjointness statements are discarded:

- ?s a cls. ?s $\mathrm{p}$ ?o . if $\mathrm{cls}$ and domain of $\mathrm{p}$ are disjoint

- ?o a cls . ?s $p$ ?o . if $\mathrm{cls}$ and range of $\mathrm{p}$ are disjoint

- ?s p1 ?o1 . ?s p2 ?०2 . if domain of $\mathrm{p} 1$ and $\mathrm{p} 2$ are disjoint

- ?s1 p1 ?० . ?s2 p2 ?० . if range of $p 1$ and $p 2$ are disjoint

- ?s p1 ?o. ?s p2 ?० . if p1 and $\mathrm{p} 2$ are disjoint

Due to lack of explicit disjointness statements in many knowledge bases, we (heuristically) assume that classes and properties that are not related via subsumption hierarchy are disjoint.

Although semantic pruning drastically reduces the amount of queries, it often does not result in only one query. HAWK thus requires a final ranking step before sending the SPARQL query to the target triple store.

\subsection{Ranking}

HAWK ranks queries using supervised training based on the gold standard answer set from the QALD-4 benchmark. In the training phase, all generated queries are run against the underlying SPARQL endpoint. Comparing the results to the gold standard answer set, HAWK stores all queries resulting with the same high F-measure. Afterwards the stored queries are used to calculate an average feature vector. HAWK's ranking calculation comprises the following components:

- NR_OF_TERMS calculates the number of nodes used to form the full-text query part as described in Section 2.6.

- NR_OF_CONSTRAINTS counts the amount of triple patterns per SPARQL query.

- NR_OF_TYPES sums the amount of patterns of the form ?var rdf: type cls.

- PREDICATES generates a vector containing an entry for each predicate used in the SPARQL query.

While running the test phase of HAWK, the cosine similarity between each SPARQL query using the above mentioned features and the average feature vector of training queries is calculated. Moreover, HAWK determines the target cardinality $x$, i.e., LIMIT $\mathrm{x}$, of each query using the indicated cardinality of the first seen POS-tag of the input query. The performance of this ranking approach is evaluated in Section 3. 


\section{Evaluation}

\subsection{Benchmark}

We evaluate HAWK against the QALD [20] benchmark. QALD has been used widely to evaluate question answering systems, e.g., TBSL, SINA, FREyA or QAKiS, which are presented above. In the recent fourth installment of QALD, hybrid questions on structured and unstructured data became a part of the benchmark. To evaluate HAWK, we focus on this hybrid training dataset comprising 25 questions, 17 out of which are entity searches using only DBpedia type information and no aggregation process. Before evaluation, we had to curate the benchmark datasets regarding, among others, incorrect grammar, typological errors, duplicate resources in the answer set. The cleaned datasets can be found in our source code repository. ${ }^{4}$

\subsection{Influence of the Entity Annotation System}

First, we evaluated the influence of the applied entity annotation system to the overall ability to produce correct answers. Thus, HAWK has been run using DBpedia Spotlight, TagMe, Fox and Wikipedia Miner. Additionally, an optimal entity annotator derived from the gold standard as well as an union of all entity annotation results was analysed.

Our results suggest that HAWK is able to retrieve correct answers with an f-measure of 0.68 using FOX as entity annotation system and assuming an optimal ranking. Furthermore, the optimal ranker is only able to achieve an F-measure of 0.58 since HAWK can cope better with missing annotation results and is tuned towards retrieving fulltext information. Against intuition, the union annotator is the worst annotation system. Merging all annotation results in queries consisting solely of semantic resources eliminating the possibility to match ontology properties and classes to important parts of the query. Thus, the Union annotator achieves only an f-measure of $0.10 .^{5}$

\subsection{Influence of the Ranking Method}

Next, evaluating the effectiveness of the feature-based ranking has to include an indepth analysis of the contribution of each feature to the overall result. Thus, we calculated the power set of the set of features and evaluated each feature group using the $\mathrm{f}$-measure reached by the top n queries. Figures 4 and 5 show the f-measure $@ N$ for result sets of size $N$.

Delving deeper into this analysis, we find:

- Although NR_OF_TERMS produces the largest sum of f-measures as a single feature, NR_OF_CONSTRAINTS achieves a higher f-measure as soon as $N=7$ due to the larger number of needed constraints with respect to the query length.

- The highest mass of f-measure reaches the pair PREDICATES, NR_OF_TERMS with an f-measure of 0.58 at $N=10$. However, HAWK is able to achieve a higher f-measure of 0.61 at $N=10$ using NR_OF_TERMS, NR_OF_CONSTRAINTS .

\footnotetext{
${ }^{4}$ https://github.com/AKSW/hawk/tree/master/resources

${ }^{5}$ Details on this evaluation can be found in the supplement on our project homepage.
} 


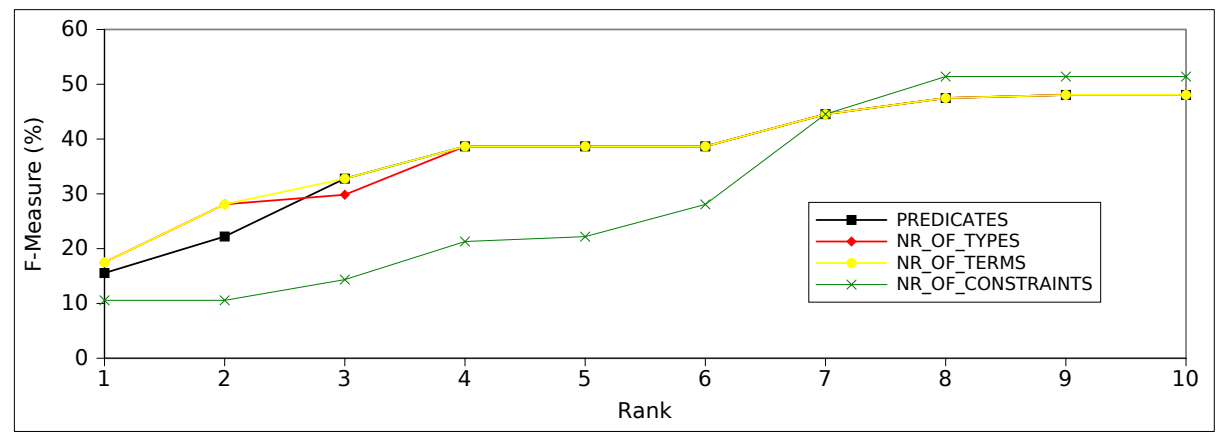

Fig. 4: F-measures on training dataset using $N=[1, \ldots, 10]$ and one feature.

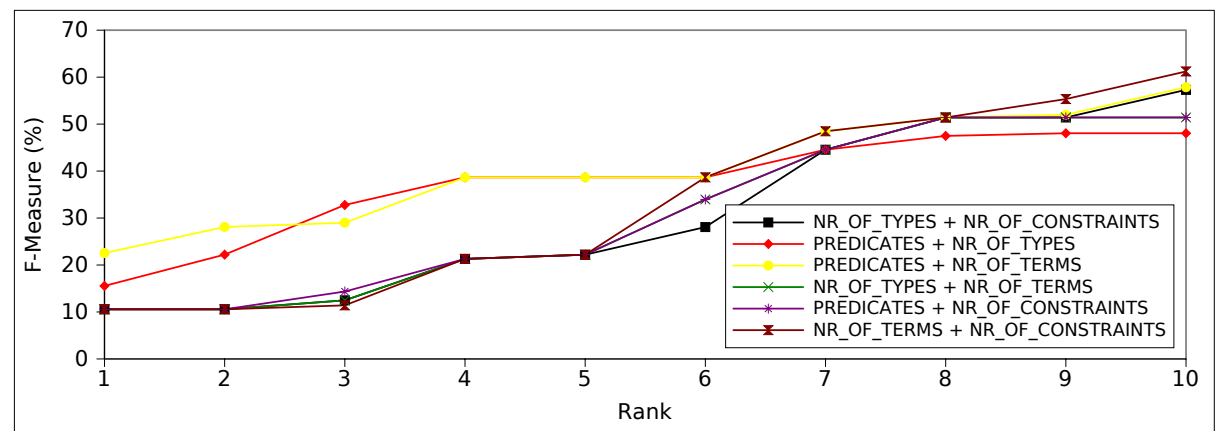

Fig. 5: F-measures on training dataset using $N=[1, \ldots, 10]$ and two features.

- The combination of three or all four features does not lead to an improvement of the f-measure.

We consider ranking the resulting SPARQL queries most challenging with respect to the fact that an ideal ranking can lead to F-measures up to 0.72 at $N=1$.

\subsection{Error Analysis}

In the following, we analyze error sources in HAWK based on the training queries failing to reach a higher F-measure. Table 4 shows for each entity search question from the training dataset its evaluation results.

- Entity Annotation: Queries 1, 11 and 15 cannot be answered by HAWK due to failing entity annotation. None of the tested annotation tools was able to either find the resources Jane_T._Austion nor G8 or Los_Alamos. Without matching entity annotations a full-text search retrieves too many matches for reaching high precision values on limited result set.

- Missing type information: some of the resources of the gold standard do not have appropriate type information leading to a high amount of queries that need to be ranked correctly. 
- Query structure: Queries like 11 or 15 inherit complex query structures leading to a multitude of interpretations while generating the SPARQL query graph.

\begin{tabular}{|c|c|c|c|c|}
\hline ID & Question & F-measure & Precision & Recall \\
\hline 1 & Give me the currencies of all G8 countries. & 0.0 & 0.0 & 0.0 \\
\hline 2 & $\begin{array}{l}\text { In which city was the assassin of Martin Luther King } \\
\text { born? }\end{array}$ & 1.0 & 1.0 & 1.0 \\
\hline 3 & $\begin{array}{l}\text { Which anti-apartheid activist graduated from the Uni- } \\
\text { versity of South Africa? }\end{array}$ & 1.0 & 1.0 & 1.0 \\
\hline 5 & $\begin{array}{l}\text { Which recipients of the Victoria Cross died in the } \\
\text { Battle of Arnhem? }\end{array}$ & 0.8 & 0.67 & 1.0 \\
\hline 6 & Where did the first man in space die? & 1.0 & 1.0 & 1.0 \\
\hline 8 & $\begin{array}{l}\text { Which members of the Wu-Tang Clan took their stage } \\
\text { name from a movie? }\end{array}$ & 0.31 & 0.18 & 1.0 \\
\hline 9 & $\begin{array}{l}\text { Which writers had influenced the philosopher that re- } \\
\text { fused a Nobel Prize? }\end{array}$ & 0.71 & 0.56 & 1.0 \\
\hline 11 & $\begin{array}{l}\text { Who composed the music for the film that depicts the } \\
\text { early life of Jane Austin? }\end{array}$ & 0.0 & 0.0 & 0.0 \\
\hline 14 & Which horses did The Long Fellow ride? & 1.0 & 1.0 & 1.0 \\
\hline 15 & $\begin{array}{l}\text { Of the people that died of radiation in Los Alamos, } \\
\text { whose death was an accident? }\end{array}$ & 0.67 & 1.0 & 0.5 \\
\hline 16 & $\begin{array}{l}\text { Which buildings owned by the crown overlook the } \\
\text { North Sea? }\end{array}$ & 0.25 & 0.14 & 1.0 \\
\hline 17 & $\begin{array}{l}\text { Which buildings in art deco style did Shreve, Lamb } \\
\text { and Harmon design? }\end{array}$ & 0.5 & 0.33 & 1.0 \\
\hline 18 & $\begin{array}{l}\text { Which birds are protected under the National Parks } \\
\text { and Wildlife Act? }\end{array}$ & 1.0 & 1.0 & 1.0 \\
\hline 19 & $\begin{array}{l}\text { Which country did the first known photographer of } \\
\text { snowflakes come from? }\end{array}$ & 1.0 & 1.0 & 1.0 \\
\hline 20 & List all the battles fought by the lover of Cleopatra. & 1.0 & 1.0 & 1.0 \\
\hline 22 & $\begin{array}{l}\text { Which actress starring in the TV series Friends owns } \\
\text { the production company Coquette Productions? }\end{array}$ & 1.0 & 1.0 & 1.0 \\
\hline 23 & $\begin{array}{l}\text { Dakar is the capital of which country member of the } \\
\text { African Union? }\end{array}$ & 1.0 & 1.0 & 1.0 \\
\hline
\end{tabular}

Table 4: Micro measures: Precision=0.70 Recall=0.85 F-measure $=0.72$ at 17 queries from QALD 4 training set. Red indicates inability to generate correct query, Blue indicates missing precision and green missing recall.

\section{Related Work}

Hybrid question answering is related to the fields of hybrid search and question answering over structured data. In the following, we thus give a brief overview of the state of the art in these two areas of research. 


\subsection{Hybrid Search}

Hybrid search approaches use a combination of structured as well as unstructured data to satisfy a user's information need. Bhagdev et al. [1] describe an approach to hybrid search combining keyword searches, Semantic Web inferencing and querying. The proposed K-Search outperforms both keyword search and pure semantic search strategies. Additionally, an user study reveals the acceptance of the Hybrid Search paradigm by end users. A personalized hybrid search implementing a hotel search service as use case is presented in [23]. By combining rule-based personal knowledge inference over subjective data, such as expensive locations, and reasoning, the personalized hybrid search has been proven to return a smaller amount of data thus resulting in more precise answers. Unfortunately, the paper does not present any qualitative evaluation and it lacks source code and test data for reproducibility.

Both approaches presented above fail to answer natural-language questions. Besides keyword-based search queries, some search engines already understand natural language questions. Question answering is more difficult than keyword-based searches since retrieval algorithms need to understand complex grammatical constructs.

\subsection{Question Answering}

Schlaefer et al. [15] describe Ephyra, an open-source question answering system and its extension with factoid and list questions via semantic technologies. Using Wordnet as well as a answer type classifier to combine statistical, fuzzy models and previously developed, manually refined rules. The disadvantage of this system lies in the handcoded answer type hierarchy which prohibits its multi-lingual use.

Cimiano et al. [4] develop ORAKEL to work on structured knowledge bases. The system is capable of adjusting its natural language interface using a refinement process on unanswered questions. Using F-logic and SPARQL as transformation objects for natural language user queries it fails to make use of Semantic Web technologies such as reasoning and entity disambiguation.

Lopez et al. [11] introduce PowerAqua, another open source system, which is agnostic of the underlying yet heterogeneous sets of knowledge bases. It detects on-the-fly the needed ontologies to answer a certain question, maps the users query to Semantic Web vocabulary and composes the retrieved (fragment-)information to an answer. However, PowerAqua is outperformed by TBSL (see below) in terms of accuracy w.r.t. the state-of-the-art QALD 3 benchmark.

Damljanovic et al. [5] present FREyA to tackle ambiguity problems when using natural language interfaces. Many ontologies in the Semantic Web contain hard to map relations, e.g., questions starting with 'How long...' can be disambiguated to a time or a distance. By incorporating user feedback and syntactic analysis FREyA is able to learn the users query formulation preferences increasing the systems question answering precision.

Cabrio et al. [2] present a demo of $Q A K i S$, an agnostic QA system grounded in ontology-relation matches. The relation matches are based on surface forms extracted from Wikipedia to enforce a wide variety of context matches, e.g., a relation birthplace(person, place) can be explicated by $\mathrm{X}$ was born in $\mathrm{Y}$ or $\mathrm{Y}$ is the birthplace of 
$\mathrm{X}$. Unfortunately, QAKiS matches only one relation per query and moreover relies on basic heuristics which do not account for the variety of natural language in general.

Unger et al. [19] describe Pythia, a question answering system based on two steps. First, it uses a domain-independent representation of a query such as verbs, determiners and wh-words. Second, Pythia is based on a domain-dependent, ontology-based interface to transform queries into F-logic. Unfortunately, Pythia does not scale for larger domains since manual mapping of ontology terms via LexInfo is required.

Moreover, Unger et al. [18] present a manually curated, template-based approach, dubbed TBSL, to match a question against a specific SPARQL query. Combining natural language processing capabilities with Linked Data leads to good benchmark results on the QALD-3 benchmark (see below). TBSL cannot be used to a wider variety of natural language questions due to its limited reportoire of 22 templates.

Shekarpour et al. [16] develop SINA a keyword and natural language query search engine which is aware of the underlying semantics of a keyword query. The system is based on Hidden Markov Models for choosing the correct dataset to query. Due to the costly Hidden Markov Models SINAs answer time (on average 3.9s) is above enduser expectations.

Treo [8] emphasis the connection between the semantic matching of input queries and the semantic distributions underlying knowledge bases. The tool is provides an entity search, a semantic relatedness measure and a search based on spreading activation.

Several industry-driven QA-related projects have emerged over the last years. For example, DeepQA of IBM Watson [7], which was able to win the Jeopardy! challenge against human experts. Further, KAIST's Exobrain ${ }^{6}$ project aims to learn from large amounts of data while ensuring a natural interaction with end users. However, it is yet limited to Korean for the moment.

For further insights please refer to $[10,12]$ which present surveys on existing question answering approaches.

\section{Conclusion}

In this paper, we presented HAWK, the first hybrid QA system for the Web of Data. We showed that by using a generic approach to generate SPARQL queries out of predicateargument structures, HAWK is able to achieve up to 0.68 F-measure on the QALD-4 benchmark. Our work on HAWK however also revealed several open research questions, of which the most important lies in finding the correct ranking approach to map a predicate-argument tree to a possible interpretation. So far, our experiments reveal that the mere finding of the right features for this endeavor remains a tedious problem. We thus aim to apply automatic feature engineering approach from deep learning in future works to automatically generate the correct ranking function. Moreover, we aim to integrate HAWK in domain-specific information systems where the more specialized context will most probably lead to higher F-measures. Finally, several components of the HAWK pipelines are computationally very complex. Finding more time-efficient algorithms for these steps will addressed in future works.

\footnotetext{
${ }^{6}$ http: //exobrain.kr/
} 


\section{Acknowledgments}

ESF Saxony and the FP7 project GeoKnow (GA No. 318159).

\section{References}

1. In The Semantic Web: Research and Applications, volume 5021 of Lecture Notes in Computer Science. 2008.

2. E. Cabrio, J. Cojan, F. Gandon, and A. Hallili. Querying multilingual dbpedia with qakis. In P. Cimiano, M. Fernández, V. Lopez, S. Schlobach, and J. Völker, editors, The Semantic Web: ESWC 2013 Satellite Events - ESWC 2013 Satellite Events, Montpellier, France, May 26-30, 2013, Revised Selected Papers, volume 7955 of Lecture Notes in Computer Science, pages 194-198. Springer, 2013.

3. J. D. Choi and M. Palmer. Getting the most out of transition-based dependency parsing. In Proceedings of the 49th Annual Meeting of the Association for Computational Linguistics: Human Language Technologies: short papers-Volume 2, pages 687-692. Association for Computational Linguistics, 2011.

4. P. Cimiano, P. Haase, J. Heizmann, M. Mantel, and R. Studer. Towards portable natural language interfaces to knowledge bases - the case of the ORAKEL system. Data Knowl. Eng., 65(2):325-354, 2008.

5. D. Damljanovic, M. Agatonovic, H. Cunningham, and K. Bontcheva. Improving habitability of natural language interfaces for querying ontologies with feedback and clarification dialogues. J. Web Sem., 19:1-21, 2013.

6. P. Ferragina and U. Scaiella. Fast and Accurate Annotation of Short Texts with Wikipedia Pages. IEEE software, 2012.

7. D. A. Ferrucci, E. W. Brown, J. Chu-Carroll, J. Fan, D. Gondek, A. Kalyanpur, A. Lally, J. W. Murdock, E. Nyberg, J. M. Prager, N. Schlaefer, and C. A. Welty. Building watson: An overview of the deepqa project. AI Magazine, 31(3):59-79, 2010.

8. A. Freitas, J. G. Oliveira, E. Curry, S. O'Riain, and J. C. P. da Silva. Treo: combining entitysearch, spreading activation and semantic relatedness for querying linked data. In Proc. of 1 st Workshop on Question Answering over Linked Data (QALD-1) at the 8th Extended Semantic Web Conference (ESWC 2011), 2011.

9. D. Gerber, A.-C. Ngonga Ngomo, S. Hellmann, T. Soru, L. Bühmann, and R. Usbeck. Realtime RDF extraction from unstructured data streams. In Proceedings of ISWC, 2013.

10. O. Kolomiyets and M.-F. Moens. A survey on question answering technology from an information retrieval perspective. Inf. Sci., 181(24):5412-5434, Dec. 2011.

11. V. Lopez, M. Fernández, E. Motta, and N. Stieler. Poweraqua: Supporting users in querying and exploring the semantic web. Semantic Web, 3(3):249-265, 2012.

12. V. Lopez, V. S. Uren, M. Sabou, and E. Motta. Is question answering fit for the semantic web?: A survey. Semantic Web, 2(2):125-155, 2011.

13. P. N. Mendes, M. Jakob, A. Garcia-Silva, and C. Bizer. DBpedia Spotlight: Shedding Light on the Web of Documents. In 7th International Conference on Semantic Systems (I-Semantics), 2011.

14. D. Milne and I. H. Witten. Learning to link with wikipedia. In 17th ACM CIKM, 2008.

15. N. Schlaefer, J. Ko, J. Betteridge, G. Sautter, M. Pathak, and E. Nyberg. Semantic extensions of the ephyra qa system for trec 2007. 2007.

16. S. Shekarpour, E. Marx, A.-C. N. Ngomo, and S. Auer. Sina: Semantic interpretation of user queries for question answering on interlinked data. Web Semantics: Science, Services and Agents on the World Wide Web, 2014. 
17. R. Speck and A.-C. N. Ngomo. Ensemble learning for named entity recognition. In The Semantic Web-ISWC 2014. 2014.

18. C. Unger, L. Bühmann, J. Lehmann, A. N. Ngomo, D. Gerber, and P. Cimiano. Templatebased question answering over RDF data. In A. Mille, F. L. Gandon, J. Misselis, M. Rabinovich, and S. Staab, editors, Proceedings of the 21st World Wide Web Conference 2012, WWW 2012, Lyon, France, April 16-20, 2012, pages 639-648. ACM, 2012.

19. C. Unger and P. Cimiano. Pythia: Compositional meaning construction for ontology-based question answering on the semantic web. volume 6716 of Natural Language Processing and Information Systems: 16th International Conference on Applications of Natural Language to Information Systems, NLDB 2011, Alicante, Spain, June 28-30, 2011. Proceedings, pages 153-160. Springer, 2011.

20. C. Unger, C. Forascu, V. Lopez, A. N. Ngomo, E. Cabrio, P. Cimiano, and S. Walter. Question answering over linked data (QALD-4). In L. Cappellato, N. Ferro, M. Halvey, and W. Kraaij, editors, Working Notes for CLEF 2014 Conference, Sheffield, UK, September 15-18, 2014. , volume 1180 of CEUR Workshop Proceedings, pages 1172-1180. CEUR-WS.org, 2014.

21. R. Usbeck. Combining linked data and statistical information retrieval. In 11th Extended Semantic Web Conference, PhD Symposium. Springer, 2014.

22. R. Usbeck, A.-C. Ngonga Ngomo, S. Auer, D. Gerber, and A. Both. Agdistis - graph-based disambiguation of named entities using linked data. In International Semantic Web Conference. 2014.

23. D. Yoo. Hybrid query processing for personalized information retrieval on the semantic web. Knowledge Base Systems, 27:211-218, 2012. 